\title{
Staggered discretizations, pressure correction schemes and all speed barotropic flows
}

\author{
L. Gastaldo ${ }^{\dagger}$, R. Herbin ${ }^{\star}$, W. Kheriji ${ }^{\star \dagger}$, C. Lapuerta ${ }^{\dagger}$, J.-C. Latché ${ }^{\dagger}$ \\ * Université Aix-Marseille \\ †Institut de Radioprotection et de Sûreté Nucléaire (IRSN)
}

herbin@cmi.univ-mrs.fr, [laura.gastaldo, walid.kheriji, celine lapuerta, jean-claude.latche]@irsn.fr

\begin{abstract}
We present in this paper a class of schemes for the solution of the barotropic NavierStokes equations. These schemes work on general meshes, preserve the stability properties of the continuous problem, irrespectively of the space and time steps, and boil down, when the Mach number vanishes, to discretizations which are standard (and stable) in the incompressible framework. Finally, we show that they are able to capture solutions with shocks to the Euler equations.
\end{abstract}

Key words : barotropic Navier-Stokes, staggered discretizations. MSC2010: $65 \mathrm{M} 12$

\section{Introduction}

The problem addressed in this paper is the system of the so-called barotropic compressible Navier-Stokes equations, which reads:

$$
\begin{aligned}
& \partial_{t} \bar{\rho}+\operatorname{div}(\bar{\rho} \overline{\boldsymbol{u}})=0, \\
& \partial_{t}(\bar{\rho} \overline{\boldsymbol{u}})+\operatorname{div}(\bar{\rho} \overline{\boldsymbol{u}} \otimes \overline{\boldsymbol{u}})+\nabla \bar{p}-\operatorname{div}(\boldsymbol{\tau}(\overline{\boldsymbol{u}}))=0, \\
& \bar{\rho}=\wp(\bar{p}),
\end{aligned}
$$

where $t$ stands for the time, $\bar{\rho}, \overline{\boldsymbol{u}}$ and $\bar{p}$ are the density, velocity and pressure in the flow, and $\boldsymbol{\tau}(\overline{\boldsymbol{u}})$ stands for the shear stress tensor. The function $\wp(\cdot)$ is the equation of state used for the modelling of the particular flow at hand, which may be the actual equation of state of the fluid or may result from assumptions concerning the flow; typically, laws as $\wp(\bar{p})=\bar{p}^{1 / \gamma}$, where $\gamma>1$ is a coefficient which is specific to the considered fluid, are obtained by making the assumption that the flow is isentropic. This system of equations is posed over $\Omega \times(0, T)$, where $\Omega$ is a domain of $\mathbb{R}^{d}, d \leq 3$ supposed to be polygonal $(d=2)$ or polyhedral $(d=3)$, and the final time $T$ is finite. We suppose that the boundary of $\Omega$ is split into $\partial \Omega=\partial \Omega_{D} \cup \partial \Omega_{N}$, and we suppose that the velocity and density are prescribed on $\partial \Omega_{D}$, while Neumann boundary conditions are prescribed on $\partial \Omega_{N}$. The flow is assumed to enter the domain through $\partial \Omega_{D}$ and to leave it through $\Omega_{N}$. This system must be supplemented by initial conditions for $\bar{\rho}$ and $\overline{\boldsymbol{u}}$. 
The objective of this paper is to present a class of schemes which enjoy three essential features. First, these schemes work on quite general two and three dimensional meshes, including locally refined nonconforming (i.e. with hanging nodes) discretizations. Second, they respect the (expected) stability properties of the continuous problem at hand, irrespectively of the space and time steps: positivity of the density, conservation of mass, energy inequality. Third, they boil down, for vanishing Mach numbers, to usual stable coupled or pressure correction schemes, which means that the discretization enjoys a discrete inf-sup condition. Even if this is beyond the scope of this paper, we remark that this latter property allows a control of the pressure to be obtained through a control of its gradient; this property is used as a central argument to obtain convergence results on model problems $[8,5,6]$.

This paper is organized as follows. First, we describe the general form of the schemes (Section 2 ). Then we show how stability requirements are taken into account to design the discretization of the velocity convection term (Section 3). The final expression for the schemes is given in Section 4, and their stability properties are stated. Finally, we discuss their capability to capture solutions of the Euler equations with shocks (Section 5).

\section{The schemes: general form}

\subsection{Meshes and unknowns}

A finite volume mesh of $\Omega$ is defined by a set $\mathcal{M}$ of non-empty convex open disjoint subsets $K$ of $\Omega$ (the control volumes), such that $\bar{\Omega}=\bigcup_{K \in \mathcal{M}} \bar{K}$. We denote by $\mathcal{E}$ the set of edges (in 2D) or faces (in $3 \mathrm{D}$ ), by $\mathcal{E}(K) \subset \mathcal{E}$ the set of faces of the cell $K \in \mathcal{M}$, by $\mathcal{E}_{\text {ext }}$ and $\mathcal{E}_{\text {int }}$ the set of boundary and interior faces, respectively. The set of external faces $\mathcal{E}_{\text {ext }}$ is split in $\mathcal{E}_{N}$ and $\mathcal{E}_{D}$, which stand for the set of the faces included in $\partial \Omega_{N}$ and $\partial \Omega_{D}$, respectively. Each internal face, denoted by $\sigma \in \mathcal{E}_{\text {int }}$, is supposed to have exactly two neighboring cells, say $K, L \in \mathcal{M}$, and $\bar{K} \cap \bar{L}=\bar{\sigma}$ which we denote by $\sigma=K \mid L$. By analogy, we write $\sigma=K \mid$ ext for an external face $\sigma$ of $K$, even if this notation is somewhat incorrect, since $K$ may have more than one external face. The mesh $\mathcal{M}$ will be referred to hereafter as the "primal mesh".

The outward normal vector to a face $\sigma$ of $K$ is denoted by $\boldsymbol{n}_{K, \sigma}$. For $K \in \mathcal{M}$ and $\sigma \in \mathcal{E}$, we

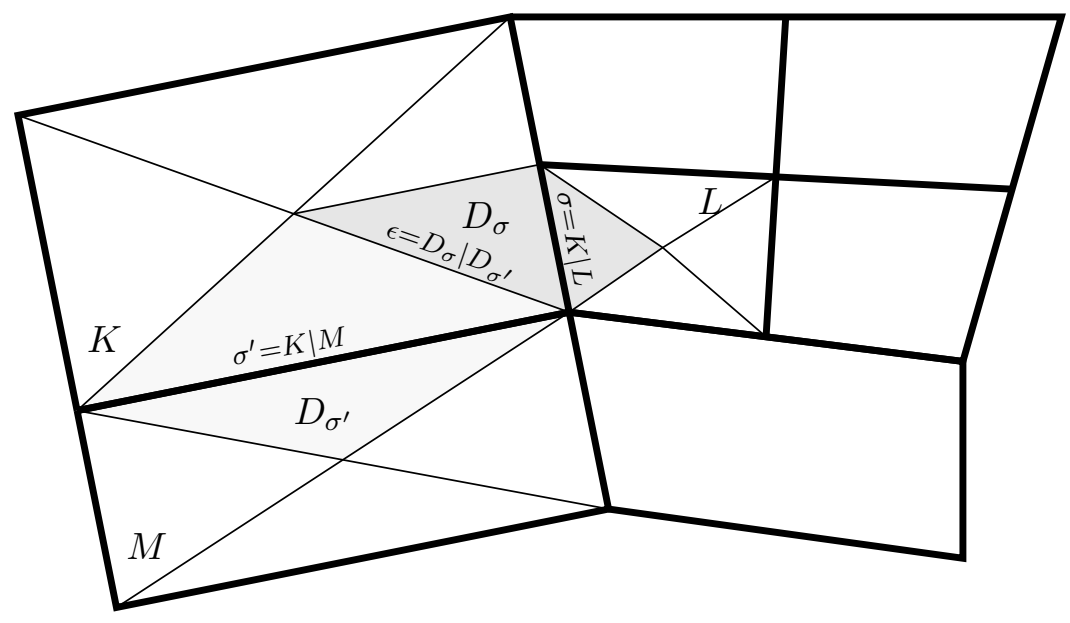

Figure 1: Notations for primal and dual cells. 
denote by $|K|$ the measure of $K$ and by $|\sigma|$ the $(d-1)$-measure of the face $\sigma$.

Then, for $\sigma \in \mathcal{E}$ and $K \in \mathcal{M}$ such that $\sigma \in \mathcal{E}(K)$ (in fact, the only cell if $\sigma \in \mathcal{E}_{\text {ext }}$ and one among the two possible cells if $\sigma \in \mathcal{E}_{\text {int }}$ ), we denote by $D_{K, \sigma}$ a subvolume of $K$ having $\sigma$ as a face (see Figure 1), and by $\left|D_{K, \sigma}\right|$ the measure of $D_{K, \sigma}$. For $\sigma \in \mathcal{E}_{\text {int }}, \sigma=K \mid L$, we set $D_{\sigma}=D_{K, \sigma} \cup D_{L, \sigma}$, so $\left|D_{\sigma}\right|=\left|D_{K, \sigma}\right|+\left|D_{L, \sigma}\right|$, and for $\sigma \in \mathcal{E}_{\text {ext }}, \sigma=K \mid$ ext, $D_{\sigma}=D_{K, \sigma}$, so $\left|D_{\sigma}\right|=\left|D_{K, \sigma}\right|$. The set of faces of the dual cell $D_{\sigma}$ is denoted by $\overline{\mathcal{E}}\left(D_{\sigma}\right)$, and the face separating two adjacent dual cells $D_{\sigma}$ and $D_{\sigma^{\prime}}$ is denoted by $\epsilon=\sigma \mid \sigma^{\prime}$.

For $1 \leq i \leq d$, the degree of freedom for the $i^{\text {th }}$ component of the velocity are assumed to be associated to a subset of $\mathcal{E}$, denoted by $\mathcal{E}^{(i)} \subset \mathcal{E}$, and are denoted by:

$$
\left\{\boldsymbol{u}_{\sigma, i}, \sigma \in \mathcal{E}^{(i)}\right\} .
$$

The sets of internal, external, Neumann and Dirichlet faces associated to the component $i$ are denoted by $\mathcal{E}_{\text {int }}^{(i)}, \mathcal{E}_{\text {ext }}^{(i)}, \mathcal{E}_{N}^{(i)}$ and $\mathcal{E}_{D}^{(i)}$ (so, for instance, $\mathcal{E}_{\text {int }}^{(i)}=\mathcal{E}_{\text {int }} \cap \mathcal{E}^{(i)}$ ). We consider the following assumption:

$$
\begin{aligned}
\text { for } 1 \leq i \leq d, \forall K \in \mathcal{M}, \\
\qquad \cup_{\sigma \in \mathcal{E}^{(i)} \cap \mathcal{E}(K)} \bar{D}_{K, \sigma}=\bar{K} \text { and } \sum_{\sigma \in \mathcal{E}^{(i)} \cap \mathcal{E}(K)}\left|D_{K, \sigma}\right|=|K|,
\end{aligned}
$$

which means that the volumes $D_{K, \sigma}, \sigma \in \mathcal{E}^{(i)}$, are disjoint, and that, for $1 \leq i \leq d,\left(D_{\sigma}\right)_{\sigma \in \mathcal{E}^{(i)}}$ is a partition of $\Omega$. The sets of faces, internal faces and Neumann faces of this dual mesh are denoted by $\overline{\mathcal{E}}^{(i)}, \overline{\mathcal{E}}_{\text {int }}^{(i)}$ and $\overline{\mathcal{E}}_{N}^{(i)}$ respectively.

We suppose that the degrees of freedom for the pressure and the density are associated to the primal cells, so they read

$$
\left\{p_{K}, K \in \mathcal{M}\right\}, \quad\left\{\rho_{K}, K \in \mathcal{M}\right\} \text {. }
$$

We denote by $\boldsymbol{V}$ the approximation space for the velocity, by $\boldsymbol{V}^{(i)}, 1 \leq i \leq d$, the approximation spaces for the velocity components and by $Q$ the approximation space for the pressure and the density, and we identify the discrete functions to their degrees of freedom:

$$
\forall \boldsymbol{v} \in \boldsymbol{V}, \boldsymbol{v}_{i} \in \boldsymbol{V}^{(i)}, 1 \leq i \leq d \text { and } \boldsymbol{v}_{i}=\left(\boldsymbol{v}_{\sigma, i}\right)_{\sigma \in \mathcal{E}^{(i)}} ; \quad \forall q \in Q, q=\left(q_{K}\right)_{K \in \mathcal{M}} .
$$

For the velocity, since the concerned degrees of freedom are located on the boundary, the Dirichlet boundary conditions are enforced in the approximation space:

$$
\text { for } 1 \leq i \leq d, \forall \boldsymbol{v}_{i} \in \boldsymbol{V}^{(i)}, \forall \sigma \in \mathcal{E}_{D}^{(i)}, \quad \boldsymbol{v}_{\sigma, i}=\frac{1}{|\sigma|} \int_{\sigma} \overline{\boldsymbol{u}}_{D, i} \mathrm{~d} \gamma,
$$

where $\overline{\boldsymbol{u}}_{D, i}$ stands for the $i^{t h}$ component of the prescribed velocity.

\subsection{The schemes}

We now introduce the following notations and assumptions:

- for $K \in \mathcal{M}$ and $\sigma \in \mathcal{E}(K)$, we denote by $\boldsymbol{u} \cdot \boldsymbol{n}_{K, \sigma}$ an approximation of the normal velocity to the face $\sigma$ outward $K$, 
- for $\boldsymbol{v} \in \boldsymbol{V}, 1 \leq i \leq d$ and $\sigma \in \mathcal{E}^{(i)}$, we denote by $(\operatorname{div} \tau(\boldsymbol{v}))_{\sigma}^{(i)}$ an approximation of the viscous term associated to $\sigma$ and to the component $i$, and we suppose that the following assumption is satisfied:

$$
\sum_{i=1}^{d} \sum_{\sigma \in \mathcal{E}^{(i)}}\left|D_{\sigma}\right|(\operatorname{div} \tau(\boldsymbol{v}))_{\sigma}^{(i)} \boldsymbol{v}_{\sigma, i} \geq 0
$$

- for $q \in Q, 1 \leq i \leq d$ and $\sigma \in \mathcal{E}^{(i)}$, we denote by $(\nabla q)_{\sigma}^{(i)}$ the component $i$ of the discrete gradient of $q$ at the face $\sigma$, and we suppose that the following assumption is satisfied for any $q \in Q$ and $\boldsymbol{v} \in \boldsymbol{V}$ :

$$
\sum_{i=1}^{d} \sum_{\sigma \in \mathcal{E}^{(i)}}\left|D_{\sigma}\right|(\nabla q)_{\sigma}^{(i)} \boldsymbol{v}_{\sigma, i}=\sum_{K \in \mathcal{M}} q_{K} \sum_{\sigma \in \mathcal{E}(K)}|\sigma| \boldsymbol{v} \cdot \boldsymbol{n}_{K, \sigma} .
$$

With these notations, we are able to write the general form of the implicit scheme:

$$
\forall K \in \mathcal{M}, \quad \frac{|K|}{\delta t}\left(\rho_{K}-\rho_{K}^{*}\right)+\sum_{\sigma \in \mathcal{E}(K)} F_{K, \sigma}=0 .
$$

For $1 \leq i \leq d, \forall \sigma \in \mathcal{E}_{\text {int }}^{(i)} \cup \mathcal{E}_{N}^{(i)}$

$$
\begin{aligned}
\frac{\left|D_{\sigma}\right|}{\delta t}\left(\rho_{\sigma} \boldsymbol{u}_{\sigma, i}-\right. & \left.\rho_{\sigma}^{*} \boldsymbol{u}_{\sigma, i}^{*}\right)+\sum_{\epsilon \in \overline{\mathcal{E}}\left(D_{\sigma}\right)} F_{\sigma, \epsilon} \boldsymbol{u}_{\epsilon, i} \\
& +\left|D_{\sigma}\right|(\boldsymbol{\nabla} p)_{\sigma}^{(i)}+\left|D_{\sigma}\right|(\operatorname{div} \tau(\boldsymbol{u}))_{\sigma}^{(i)}=0
\end{aligned}
$$

$$
\forall K \in \mathcal{M}, \quad \rho_{K}=\wp\left(p_{K}\right),
$$

where the ${ }^{*}$ superscript denotes the beginning-of-step quantities, $F_{K, \sigma}$ stands for the mass flux leaving $K$ through $\sigma, \rho_{\sigma}$ stands for an approximation of the density at the face, and $F_{\sigma, \epsilon}$ is a mass flux leaving $D_{\sigma}$ through $\epsilon$. For the flux $F_{K, \sigma}$ at the internal face $\sigma=K \mid L$, we choose an upwind approximation of the density:

$$
F_{K, \sigma}=|\sigma| \boldsymbol{u} \cdot \boldsymbol{n}_{K, \sigma} \rho_{\sigma}^{\text {up }}, \quad \text { with } \rho_{\sigma}^{\text {up }}=\rho_{K} \text { if } F_{K, \sigma} \geq 0, \rho_{\sigma}^{\text {up }}=\rho_{L} \text { otherwise. }
$$

On $\sigma \in \mathcal{E}_{D}$, the density $\rho_{\sigma}^{\text {up }}$ is given by the boundary condition, and, on $\sigma \in \mathcal{E}_{N}, \sigma=K \mid$ ext, $\rho_{\sigma}^{\text {up }}=\rho_{K}$, which is indeed an upwind choice, since the flow is supposed to enter the domain through $\partial \Omega_{D}$ and to leave it through $\partial \Omega_{N}$. For the velocity components at the dual faces, $\boldsymbol{u}_{\epsilon, i}$, we choose either the centred or upwind approximation on the internal faces, and the value at the face for the outflow faces.

A pressure correction scheme is obtained from (2) by splitting the resolution in two steps:

1- Velocity prediction step - Solve for $\tilde{\boldsymbol{u}} \in \boldsymbol{V}$ the momentum balance equation with the beginning-of-step pressure:

For $1 \leq i \leq d, \forall \sigma \in \mathcal{E}_{\text {int }}^{(i)} \cup \mathcal{E}_{N}^{(i)}$

$$
\begin{aligned}
\frac{\left|D_{\sigma}\right|}{\delta t}\left(\rho_{\sigma} \tilde{\boldsymbol{u}}_{\sigma, i}-\right. & \left.\rho_{\sigma}^{*} \boldsymbol{u}_{\sigma, i}^{*}\right)+\sum_{\epsilon \in \overline{\mathcal{E}}\left(D_{\sigma}\right)} F_{\sigma, \epsilon} \tilde{\boldsymbol{u}}_{\epsilon, i} \\
& +\left|D_{\sigma}\right|\left(\boldsymbol{\nabla} p^{*}\right)_{\sigma}^{(i)}+\left|D_{\sigma}\right|(\operatorname{div} \tau(\tilde{\boldsymbol{u}}))_{\sigma}^{(i)}=0,
\end{aligned}
$$


2 - $\quad$ Correction step - Solve for $\boldsymbol{u} \in \boldsymbol{V}$ and $p \in Q$ :

$\forall K \in \mathcal{M}, \quad \frac{|K|}{\delta t}\left(\rho_{K}-\rho_{K}^{*}\right)+\sum_{\sigma \in \mathcal{E}(K)} F_{K, \sigma}=0$.

For $1 \leq i \leq d, \forall \sigma \in \mathcal{E}_{\text {int }}^{(i)} \cup \mathcal{E}_{N}^{(i)}$

$$
\frac{\left|D_{\sigma}\right|}{\delta t} \rho_{\sigma}\left(\boldsymbol{u}_{\sigma, i}-\tilde{\boldsymbol{u}}_{\sigma, i}\right)+\left|D_{\sigma}\right|\left(\boldsymbol{\nabla}\left(p-p^{*}\right)\right)_{\sigma}^{(i)}=0
$$

$\forall K \in \mathcal{M}, \quad \rho_{K}=\wp\left(p_{K}\right)$.

The equations of the correction step are combined to produce a nonlinear parabolic problem for the pressure, which reads, $\forall K \in \mathcal{M}$ :

$$
\begin{aligned}
\frac{|K|}{\delta t}\left(\wp\left(p_{K}\right)-\rho_{K}^{*}\right)+\sum_{\sigma=K \mid L} \frac{\rho_{\sigma}^{\text {up }}}{\rho_{\sigma}} \frac{|\sigma|^{2}}{\left|D_{\sigma}\right|}\left(\phi_{K}-\phi_{L}\right) & +\sum_{\sigma \in \mathcal{E}(K) \cap \mathcal{E}_{N}} \frac{\rho_{\sigma}^{\mathrm{up}}}{\rho_{\sigma}} \frac{|\sigma|^{2}}{\left|D_{\sigma}\right|} \phi_{K} \\
& =\frac{1}{\delta t} \sum_{\sigma \in \mathcal{E}(K)}|\sigma| \rho_{\sigma}^{\mathrm{up}} \tilde{\boldsymbol{u}} \cdot \boldsymbol{n}_{K, \sigma},
\end{aligned}
$$

where $\phi \in Q$ is defined by $\phi=p-p^{*}$. Note that the second and third terms at the left-hand side look like a finite volume discretization of a diffusion operator, with homogeneous Neumann boundary conditions on $\mathcal{E}_{D}$ and Dirichlet boundary conditions on $\mathcal{E}_{N}$ for the pressure increment, as usual in pressure correction schemes (see [4] for a discussion on the effects of these spurious boundary conditions).

The standard discretizations entering the present framework are either low-degree nonconforming finite elements, namely the Crouzeix-Raviart element [3] for simplicial meshes or the Rannacher-Turek element [23] for quadrangles and hexahedra, or, for structured cartesian grids, the MAC scheme $[13,14]$. We describe here the construction of the diffusion and pressure gradient terms for the finite element schemes, supposing for short that the velocity obeys homogeneous Dirichlet boundary conditions on $\partial \Omega$. Let $\sigma \in \mathcal{E}_{\text {int }}$ and $\varphi_{\sigma}$ be the finite element shape function associated to $\sigma$. In Rannacher-Turek or Crouzeix-Raviart elements, a degree of freedom for each component of the velocity is associated to each face, so $\mathcal{E}_{\text {int }}^{(i)}=\mathcal{E}_{\text {int }}$, for $1 \leq i \leq d$. Let $1 \leq i \leq d$ be given, let $\boldsymbol{e}^{(i)}$ be the $i^{\text {th }}$ vector of the canonical basis of $\mathbb{R}^{d}$ and let us define $\boldsymbol{\varphi}_{\sigma}^{(i)}$ by:

$$
\varphi_{\sigma}^{(i)}=\varphi_{\sigma} e^{(i)}
$$

Then the usual finite element discretization of the diffusion term reads, for a constant viscosity Newtonian fluid (that is supposing $\operatorname{div} \boldsymbol{\tau}(\boldsymbol{u})=\mu \Delta \boldsymbol{u}+(\mu / 3) \boldsymbol{\nabla} \operatorname{div}(\boldsymbol{u})$, with $\mu$ the viscosity):

$$
\left|D_{\sigma}\right|(\operatorname{div} \boldsymbol{\tau}(\boldsymbol{u}))_{\sigma}^{(i)}=\sum_{K \in \mathcal{M}} \mu \int_{K} \boldsymbol{\nabla} \boldsymbol{u}: \nabla \boldsymbol{\varphi}_{\sigma}^{(i)} \mathrm{d} \boldsymbol{x}+\frac{\mu}{3} \int_{K} \operatorname{div} \boldsymbol{u} \operatorname{div} \boldsymbol{\varphi}_{\sigma}^{(i)} \mathrm{d} \boldsymbol{x}
$$

The pressure gradient term at the internal face $\sigma=K \mid L$ reads:

$$
\left|D_{\sigma}\right|(\nabla p)_{\sigma}^{(i)}=\sum_{K \in \mathcal{M}} \int_{K} p \operatorname{div} \boldsymbol{\varphi}_{\sigma}^{(i)} \mathrm{d} \boldsymbol{x}=|\sigma|\left(p_{L}-p_{K}\right) \boldsymbol{n}_{K, \sigma} \cdot \boldsymbol{e}^{(i)} .
$$




\section{The stability issue and consequences}

\subsection{A stability result for the convection}

At the continuous level, let us assume that the mass balance $\partial_{t} \rho+\operatorname{div}(\boldsymbol{\beta})=0$ holds, with $\boldsymbol{\beta}$ a regular vector-valued function. Then, for all scalar regular functions $u$ and $v$, we have:

$$
\begin{aligned}
\int_{\Omega}\left[\partial_{t}(\rho u)+\operatorname{div}(u \boldsymbol{\beta})\right] v \mathrm{~d} \boldsymbol{x}= & \\
& \int_{\Omega}\left[\partial_{t}(\rho u)-\frac{1}{2}\left(\partial_{t} \rho\right) u\right] v \mathrm{~d} \boldsymbol{x}+s(u, v)+\frac{1}{2} \int_{\partial \Omega} u v \boldsymbol{\beta} \cdot \boldsymbol{n} \mathrm{d} \gamma
\end{aligned}
$$

where $s$ is the following skew-symmetric bilinear form:

$$
s(u, v)=\frac{1}{2} \int_{\Omega} v \boldsymbol{\beta} \cdot \nabla u \mathrm{~d} \boldsymbol{x}-\frac{1}{2} \int_{\Omega} u \boldsymbol{\beta} \cdot \nabla v \mathrm{~d} \boldsymbol{x} .
$$

Taking $u=v=\boldsymbol{u}_{i}$ and summing over $i$, the first term gives the time derivative of the kinetic energy, the second term vanishes and the last term corresponds to the kinetic energy flux through the boundary of the domain. The following Lemma, proven in [20], states a discrete counterpart of this computation in the case where the (possible) Dirichlet boundary conditions are homogeneous (see also [1] and [9] for a direct estimate of the kinetic energy, for an implicit and explicit scheme respectively).

Lemma 3.1 Let us suppose that, for an index $i, 1 \leq i \leq d$, the following discrete mass balance holds over the dual cells associated to the $i^{\text {th }}$ component of the velocity:

$$
\forall \sigma \in \mathcal{E}_{\text {int }}^{(i)} \cup \mathcal{E}_{N}^{(i)}, \quad \frac{\left|D_{\sigma}\right|}{\delta t}\left(\rho_{\sigma}-\rho_{\sigma}^{*}\right)+\sum_{\epsilon \in \overline{\mathcal{E}}\left(D_{\sigma}\right)} F_{\sigma, \epsilon}=0 .
$$

Let $u, v \in V^{(i)}$, and let us suppose that these discrete functions obey homogeneous Dirichlet boundary. Then we have:

$$
\begin{aligned}
& \sum_{\sigma \in \mathcal{E}_{\text {int }}^{(i)} \cup \mathcal{E}_{N}^{(i)}} v_{\sigma}\left[\frac{\left|D_{\sigma}\right|}{\delta t}\left(\rho_{\sigma} u_{\sigma}-\rho_{\sigma}^{*} u_{\sigma}^{*}\right)+\sum_{\epsilon \in \overline{\mathcal{E}}\left(D_{\sigma}\right)} F_{\sigma, \epsilon} u_{\epsilon}\right] \\
& \geq T_{\Omega, \mathrm{k}}(u, v)+T_{\Omega, \mathrm{s}}(u, v)+T_{\partial \Omega}(u, v)
\end{aligned}
$$

with:

$$
\begin{aligned}
& T_{\Omega, \mathrm{k}}(u, v)=\sum_{\sigma \in \mathcal{E}_{\mathrm{int}}^{(i)} \cup \mathcal{E}_{N}^{(i)}} \frac{\left|D_{\sigma}\right|}{\delta t}\left(\rho_{\sigma} u_{\sigma}-\rho_{\sigma}^{*} u_{\sigma}^{*}\right) v_{\sigma}-\frac{1}{2}\left(\rho_{\sigma}-\rho_{\sigma}^{*}\right) u_{\sigma} v_{\sigma}, \\
& T_{\Omega, \mathrm{s}}(u, v)=S(u, v)-S(v, u), \quad S(u, v)=\frac{1}{2} \sum_{\epsilon \in \overline{\mathcal{E}}_{\mathrm{int}}^{(i)}, \epsilon=D_{\sigma} \mid D_{\sigma^{\prime}}} F_{\sigma, \epsilon} v_{\epsilon}\left(u_{\sigma^{\prime}}-u_{\sigma}\right), \\
& T_{\partial \Omega}(u, v)=\frac{1}{2} \sum_{\epsilon \in \overline{\mathcal{E}}_{N}^{(i)}, \sigma=D_{\sigma} \mid \mathrm{ext}} F_{\sigma, \epsilon} u_{\epsilon} v_{\epsilon} .
\end{aligned}
$$

Inequality (9) becomes an equality for a centred choice of the discretization of the face values $u_{\epsilon}$. 
Of course, $T_{\Omega, \mathrm{s}}(u, u)=0$, and an easy computation shows that:

$$
T_{\Omega, \mathrm{k}}(u, u) \geq \frac{1}{2 \delta t} \sum_{\sigma \in \mathcal{E}_{\mathrm{int}}^{(i)} \cup \mathcal{E}_{N}^{(i)}}\left|D_{\sigma}\right|\left[\rho_{\sigma} u_{\sigma}^{2}-\rho_{\sigma}^{*}\left(u_{\sigma}^{*}\right)^{2}\right] .
$$

Applying Lemma 3.1 to each component of the velocity, the obtained term is thus the discrete time-derivative of the kinetic energy, and may be used to obtain stability estimates for the scheme (see Section 4).

\section{Remark 1 (Non-homogeneous Dirichlet boundary conditions)}

The limitation to homogeneous Dirichlet boundary conditions may be seen, from the proof, to stem from the fact that no balance equation is written on the dual cells associated to faces lying on $\partial \Omega_{D}$. The problem may thus be fixed by keeping these degrees of freedom and using a penalization technique.

Remark 2 (Artificial boundary conditions) Lemma 3.1 may be used to derive artificial boundary conditions allowing the flow to enter the domain through $\partial \Omega_{N}$, by first collecting the boundary terms in the variational form of the momentum balance equation (i.e. adding to $T_{\partial \Omega}(u, v)$ the terms issued from the diffusion and the pressure gradient) and then imposing that the result may be written as a linear form acting on the test function (see [2] for a similar development in the incompressible case). The so-built boundary condition is observed in practice to give quite good results when modelling external flows [20].

\subsection{Discretization of the convection term}

The problem to tackle is now the following one: on one side, the discrete mass balance over the dual cells (8) is necessary for the stability of the scheme; on the other side, the mass balance is only written by the scheme(s) for the primal cells (Equation (2a) or (5a)). We are thus lead to express the mass fluxes $\left(F_{\sigma, \epsilon}\right)$ through the dual faces as a function of the mass fluxes $\left(F_{K, \sigma}\right)$ through the primal faces, in such a way that the discrete balance over the primal cells yields a discrete balance over the dual cells. We describe in this section how this may be done, first for the MAC (structured) mesh (see also [15]) and, second, for the Rannacher-Turek element on general quadrangles.

\subsubsection{MAC scheme}

For the MAC scheme, in two space dimensions and with the local notations introduced on Figure 2 , the mass balance on the primal cells reads:

$$
\begin{array}{ll}
K: \quad & \frac{|K|}{\delta t}\left(\varrho_{K}-\varrho_{K}^{*}\right)-F_{W}-F_{S W}+F_{C}+F_{N W}=0, \\
L: & \frac{|L|}{\delta t}\left(\varrho_{L}-\varrho_{L}^{*}\right)-F_{C}-F_{S E}+F_{E}+F_{N E}=0 .
\end{array}
$$

Multiplying both equations by $1 / 2$ and summing them yields, for $\sigma=K \mid L$ :

$$
\begin{aligned}
& \frac{\left|D_{\sigma}\right|}{\delta t}\left(\varrho_{\sigma}-\varrho_{\sigma}^{*}\right) \\
& \quad-\frac{1}{2}\left[F_{W}+F_{C}\right]-\frac{1}{2}\left[F_{S W}+F_{S E}\right]+\frac{1}{2}\left[F_{C}+F_{E}\right]+\frac{1}{2}\left[F_{N W}+F_{N E}\right]=0
\end{aligned}
$$




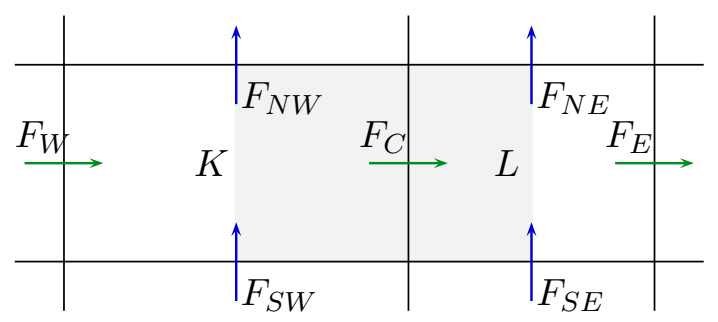

Figure 2: Local notations for the definition of the mass fluxes at the dual edges with the MAC scheme.

with the usual definition of the dual cell $D_{\sigma}$, which implies that $\left|D_{K, \sigma}\right|=|K| / 2$ and $\left|D_{L, \sigma}\right|=$ $|L| / 2$, and with the following definition of the density on the face:

$$
\left|D_{\sigma}\right| \varrho_{\sigma}=\left|D_{K, \sigma}\right| \varrho_{K}+\left|D_{L, \sigma}\right| \varrho_{L} .
$$

Equation (10) thus suggests the following definition for the mass fluxes at the dual faces:

$$
\begin{aligned}
& \text { left face: } \quad F_{\sigma, \epsilon}=-\frac{1}{2}\left[F_{W}+F_{C}\right] ; \quad \text { right face: } \quad F_{\sigma, \epsilon}=\frac{1}{2}\left[F_{C}+F_{E}\right] \text {; } \\
& \text { bottom face: } \quad F_{\sigma, \epsilon}=-\frac{1}{2}\left[F_{S W}+F_{S E}\right] ; \quad \text { top face: } \quad F_{\sigma, \epsilon}=\frac{1}{2}\left[F_{N W}+F_{N E}\right] \text {. }
\end{aligned}
$$

Note that this definition is rather non-standard: for instance, the flux at the left face of $D_{\sigma}$, which is included in $K$, may involve densities of the neighbouring primal cells. The extension of the above construction to the three-dimensional case is straightforward.

\subsubsection{Rannacher-Turek element}

A construction similar to that of the MAC scheme may be performed for rectangular meshes. For $K$ and $L$ two neighbouring cells of $\mathcal{M}$, the half-diamond cell $D_{K, \sigma}$ (resp. $D_{L, \sigma}$ ) associated to the common face $\sigma=K \mid L$ is defined as the cone with vertex the mass center of $K$ (resp. $L$ ) and with basis $\sigma$, the density $\rho_{\sigma}$ is defined by the weighted average (11), and the dual mass

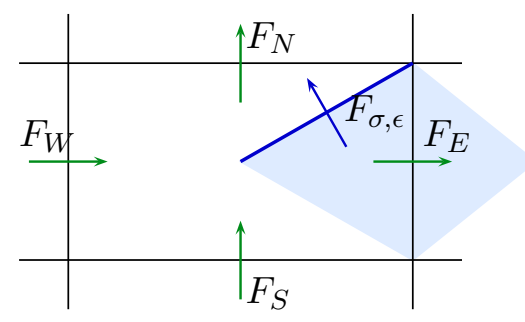

Figure 3: Local notations for the definition of the mass fluxes at the dual edges with the Rannacher-Turek element 
fluxes are obtained by multiplying the mass balances over $K$ and $L$ by $1 / 4$ and summing. With the local notations of Figure 3, this yields, for the dual mass flux $F_{\sigma, \epsilon}$, an expression of the form:

$$
F_{\sigma, \epsilon}=-\frac{1}{8} F_{W}+\frac{3}{8} F_{N}-\frac{3}{8} F_{E}+\frac{1}{8} F_{S} .
$$

We now explain how to extend this formulation to general meshes.

Let us suppose that, for any cell $K \in \mathcal{M}$, we are able to define the fluxes through the dual faces included in $K$ in such a way that:

(A1) The mass balance over the half-diamond cells is proportional to the mass balance over $K$, in the following sense:

$$
\forall \sigma \in \mathcal{E}(K), \quad F_{K, \sigma}+\sum_{\epsilon \in \overline{\mathcal{E}}\left(D_{\sigma}\right), \epsilon \subset K} F_{\sigma, \epsilon}=\xi_{K}^{\sigma} \sum_{\sigma \in \mathcal{E}(K)} F_{K, \sigma},
$$

with $\sum_{\sigma \in \mathcal{E}(K)} \xi_{K}^{\sigma}=1$ and, for any $\sigma \in \mathcal{E}(K), \xi_{K}^{\sigma} \geq 0$.

(A2) The dual fluxes are conservative, i.e., for any $\epsilon=D_{\sigma} \mid D_{\sigma}^{\prime}, F_{\sigma, \epsilon}=-F_{\sigma^{\prime}, \epsilon}$.

(A3) The dual fluxes are bounded with respect to the $\left(F_{K, \sigma}\right)_{\sigma \in \mathcal{E}(K)}$ :

$$
\forall \sigma \in \mathcal{E}(K), \forall \epsilon \in \overline{\mathcal{E}}\left(D_{\sigma}\right) \subset K \quad\left|F_{\sigma, \epsilon}\right| \leq C \max \left\{\left|F_{K, \sigma}\right|, \sigma \in \mathcal{E}(K)\right\} .
$$

In addition, let us define $\left|D_{K, \sigma}\right|$ as:

$$
\left|D_{K, \sigma}\right|=\xi_{K}^{\sigma}|K|
$$

and $\rho_{\sigma}$, once again, by the weighted average (11). Then the dual fluxes satisfy the required mass balance. Indeed, for $\sigma \in \mathcal{E}_{\text {int }}, \sigma=K \mid L$, we have:

$$
\begin{aligned}
& \frac{\left|D_{\sigma}\right|}{\delta t}\left(\rho_{\sigma}-\rho_{\sigma}^{*}\right)+\sum_{\epsilon \in \mathcal{E}\left(D_{\sigma}\right)} F_{\sigma, \epsilon} \\
& =\frac{\left|D_{K, \sigma}\right|}{\delta t}\left(\rho_{K}-\rho_{K}^{*}\right)+F_{K, \sigma}+\sum_{\epsilon \in \overline{\mathcal{E}}\left(D_{\sigma}\right), \epsilon \subset K} F_{\sigma, \epsilon} \\
& +\frac{\left|D_{L, \sigma}\right|}{\delta t}\left(\rho_{L}-\rho_{L}^{*}\right)+F_{L, \sigma}+\sum_{\epsilon \in \overline{\mathcal{E}}\left(D_{\sigma}\right), \epsilon \subset L} F_{\sigma, \epsilon} \\
& =\xi_{K}^{\sigma}\left[\frac{|K|}{\delta t}\left(\rho_{K}-\rho_{K}^{*}\right)+\sum_{\sigma \in \mathcal{E}(K)} F_{K, \sigma}\right]+\xi_{L}^{\sigma}\left[\frac{|L|}{\delta t}\left(\rho_{L}-\rho_{L}^{*}\right)+\sum_{\sigma \in \mathcal{E}(L)} F_{L, \sigma}\right]=0 .
\end{aligned}
$$

A similar computation leads to the same conclusion for the (half-)dual cells associated to the Neumann boundary faces.

The next issue is to check whether Assumptions (A1)-(A3) are sufficient for the consistency of the scheme. In this respect, the following lemma [16] brings a decisive argument.

Lemma 3.2 Let Assumptions (A1)-(A3) hold. For $v \in V$ and $K \in \mathcal{M}$, let $v_{K}$ be defined by $v_{K}=\sum_{\sigma \in \mathcal{E}(K)} \xi_{K}^{\sigma} v_{\sigma}$. Let $u \in V$, and $R(u, v)$ be the quantity defined by:

$$
R(u, v)=\sum_{\sigma \in \mathcal{E}_{\text {int }}} v_{\sigma} \sum_{\substack{\epsilon \in \overline{\mathcal{E}}\left(D_{\sigma}\right), \epsilon=D_{\sigma} \mid D_{\sigma}^{\prime}}} F_{\sigma, \epsilon} \frac{u_{\sigma}+u_{\sigma^{\prime}}}{2}-\sum_{K \in \mathcal{M}} v_{K} \sum_{\sigma \in \mathcal{E}(K)} F_{K, \sigma} u_{\sigma} .
$$


Let us suppose that the primal fluxes are associated to a convection momentum field $\boldsymbol{\beta}$, i.e. $\forall K \in \mathcal{M}, \forall \sigma \in \mathcal{E}(K), \quad F_{K, \sigma}=|\sigma| \boldsymbol{\beta}_{\sigma} \cdot \boldsymbol{n}_{K, \sigma}$. (For the schemes used here, of course, $\boldsymbol{\beta}$ depends the density and the velocity, see (3).) Then there exists $C$ depending only on the regularity of the mesh such that:

$$
|R(u, v)| \leq C h\|\boldsymbol{\beta}\|_{l^{\infty}}\|u\|_{1}\|v\|_{1},
$$

with $\|\boldsymbol{\beta}\|_{l^{\infty}}=\max _{\sigma \in \mathcal{E}}\left|\boldsymbol{\beta}_{\sigma}\right|$ and the discrete $\mathrm{H}^{1}$-norm on the dual mesh is defined by:

$$
\forall v \in V, \quad\|v\|_{1}=\sum_{K \in \mathcal{M}} h_{K}^{d-2} \sum_{\sigma, \sigma^{\prime} \in \mathcal{E}(K)}\left(v_{\sigma}-v_{\sigma^{\prime}}\right)^{2} .
$$

The quantity $R(u, v)$ compares two discrete analogues to $\int_{\Omega} v \operatorname{div}(u \boldsymbol{\beta}) \mathrm{d} \boldsymbol{x}$; the first analogue is defined with the divergence taken over the dual meshes while the second analogue is defined with the divergence over the primal cells. Let us suppose that the discrete $\mathrm{H}^{1}$-norm of the solution is controlled thanks to the diffusion term. Then, in a convergence or error analysis study in the linear case (i.e. with a given regular convection field $\boldsymbol{\beta}$ ), Lemma 3.2 allows to replace the first discrete analogue by the second one, thus substituting well defined quantities to quantities only defined through (A1)-(A3). It is used in [16] to prove that the scheme is first-order for the stationary convection-diffusion equation. The convergence for the constant density NavierStokes equations (that is with $\boldsymbol{\beta}=\boldsymbol{u}$ ) was also proven, controlling now $\|\boldsymbol{u}\|_{l^{\infty}}$ by $\|\boldsymbol{u}\|_{1}$ thanks to an inverse inequality.

The last task is now to build fluxes satisfying (A1)-(A3); this is easily done by choosing $\xi_{K}^{\sigma}=1 / 4$, and keeping for the expression of the dual fluxes as a function of the primal fluxes the same linear combination (12) as in the rectangular case. Note that this implicitly implies that the geometrical definition of the dual cells has been generalized, since it is not possible in general to split a quadrangle in four simplices of same measure (even if the quadrangle is convex) . The extension to three dimensions only needs to deal with the rectangular parallelepipedic case, which is quite simple [1]. Finding directly a solution to (A1)-(A3) may also be an alternative route, to deal with more complex cases, as done in [16] to extend the scheme to locally refined nonconforming grids.

\section{Schemes and stability estimates}

In order to obtain the complete formulation of the considered schemes, we now have to fix the time-marching procedure. This is straightforward for the implicit scheme, and we concentrate here on the pressure correction scheme. The problem which we face in this case is that the mass balance is not yet solved when performing the prediction step. In our implementations in the ISIS computer code [18] developed at IRSN on the basis of the software component library PELICANS [22], it is circumvented by just shifting in time the density $\rho_{\sigma}$; the mass balance on the dual cells is recovered from the mass balance on the primal cells at the previous time step. This has essentially two drawbacks. First, the trick indeed works only if the time step is constant; for a variable time step, one has to choose between loosing stability or consistency (locally in time, so fortunately, without observed impact in practice). Second, the scheme is only first order in time.

In addition, stability seems to require an initial pressure renormalization step, which is an algebraic variant of the one introduced in [12]. It seems however that this step may be omitted in practice. 
The algorithm (keeping in this presentation the pressure renormalization step) reads, assuming that $\boldsymbol{u}^{n}, p^{n}, \rho^{n}$ and the family $\left(F_{K, \sigma}^{n}\right)$ are known:

1- Pressure renormalization step - Let $\left(\lambda_{\sigma}\right)_{\sigma \in \mathcal{E}_{\text {int }}}$ be a family of positive real numbers, and let $-\operatorname{div}(\lambda \boldsymbol{\nabla})_{\mathcal{M}}$ be the discrete elliptic operator from $Q$ to $Q$ defined by, $\forall K \in \mathcal{M}$ and $q \in Q:$

$$
\left[-\operatorname{div}(\lambda \boldsymbol{\nabla})_{\mathcal{M}}(q)\right]_{K}=\sum_{\sigma=K \mid L} \lambda_{\sigma} \frac{|\sigma|^{2}}{\left|D_{\sigma}\right|}\left(q_{K}-q_{L}\right)+\sum_{\sigma \in \mathcal{E}_{N}, \sigma=K \mid \operatorname{ext}} \lambda_{\sigma} \frac{|\sigma|^{2}}{\left|D_{\sigma}\right|} q_{K} .
$$

Then $\tilde{p}^{n+1} \in Q$ is given by:

$$
-\operatorname{div}\left(\frac{1}{\rho^{n}} \nabla\right)_{\mathcal{M}}\left(\tilde{p}^{n+1}\right)=-\operatorname{div}\left(\frac{1}{\left[\rho^{n} \rho^{n-1}\right]^{1 / 2}} \nabla\right)_{\mathcal{M}}\left(p^{n}\right),
$$

the weights $\left(\rho_{\sigma}^{n}\right)_{\sigma \in \mathcal{E}_{\text {int }} \cup \mathcal{E}_{N}}$ and $\left(\rho_{\sigma}^{n-1}\right)_{\sigma \in \mathcal{E}_{\text {int }} \cup \mathcal{E}_{N}}$ being the densities involved in the timederivative term of the momentum balance equation.

2- $\quad$ Velocity prediction step - Solve for $\tilde{\boldsymbol{u}}^{n+1} \in \boldsymbol{V}$, for $1 \leq i \leq d$ and $\forall \sigma \in \mathcal{E}_{\text {int }}^{(i)} \cup \mathcal{E}_{N}^{(i)}$ :

$$
\begin{aligned}
\frac{\left|D_{\sigma}\right|}{\delta t}\left(\rho_{\sigma}^{n} \tilde{\boldsymbol{u}}_{\sigma, i}^{n+1}-\rho_{\sigma}^{n-1} \boldsymbol{u}_{\sigma, i}^{n}\right)+\sum_{\epsilon \in \overline{\mathcal{E}}\left(D_{\sigma}\right)} & F_{\sigma, \epsilon}^{n} \tilde{\boldsymbol{u}}_{\epsilon, i}^{n+1} \\
& +\left|D_{\sigma}\right|\left(\boldsymbol{\nabla} \tilde{p}^{n+1}\right)_{\sigma}^{(i)}+\left|D_{\sigma}\right|\left(\operatorname{div} \tau\left(\tilde{\boldsymbol{u}}^{n+1}\right)\right)_{\sigma}^{(i)}=0
\end{aligned}
$$

where the $\left(F_{\sigma, \epsilon}^{n}\right)_{\epsilon \in \overline{\mathcal{E}}\left(D_{\sigma}\right)}$ are built as explained in the previous section, from the primal fluxes at time $t^{n}$.

3 - Correction step - Solve for $\boldsymbol{u}^{n+1} \in \boldsymbol{V}$ and $p^{n+1} \in Q$ :

$$
\begin{aligned}
& \forall K \in \mathcal{M}, \quad \frac{|K|}{\delta t}\left(\rho_{K}^{n+1}-\rho_{K}^{n}\right)+\sum_{\sigma \in \mathcal{E}(K)} F_{K, \sigma}^{n+1}=0 . \\
& \text { For } 1 \leq i \leq d, \forall \sigma \in \mathcal{E}_{\text {int }}^{(i)} \cup \mathcal{E}_{N}^{(i)}, \\
& \qquad \frac{\left|D_{\sigma}\right|}{\delta t} \rho_{\sigma}^{n}\left(\boldsymbol{u}_{\sigma, i}^{n+1}-\tilde{\boldsymbol{u}}_{\sigma, i}^{n+1}\right)+\left|D_{\sigma}\right|\left(\nabla\left(p^{n+1}-\tilde{p}^{n+1}\right)\right)_{\sigma}^{(i)}=0, \\
& \forall K \in \mathcal{M}, \quad \rho_{K}^{n+1}=\wp\left(p_{K}^{n+1}\right) .
\end{aligned}
$$

The algorithm must be initialized by the data $\boldsymbol{u}^{0} \in \boldsymbol{V}, \rho^{-1} \in Q$ and $\rho^{0} \in Q$ satisfying the discrete mass balance equation, and with the corresponding mass fluxes $\left(F_{K, \sigma}^{0}\right)$. A possible way to obtain these quantities is to evaluate $\boldsymbol{u}^{0}$ and $\rho^{-1}$ from the initial conditions, and, as a preliminary step, to solve for $\rho^{0}$ the mass balance equation.

The upwinding in the discretization of the mass balance equation has for consequence that any density appearing in the algorithm is positive (provided that the initial density is positive). The existence and uniqueness of a solution to Steps 1 and 2 is then clear: these are linear problems with coercive operators (for Step 2, thanks to the stability of the convection term). The existence of a solution to Step 3 may be obtained by a Brouwer fixed point argument, using the fact that 
the conservativity of the mass balance yields an estimate for $\rho$, so for $p$, and finally for $\boldsymbol{u}$ (in any norm, since we work on finite dimensional spaces). The algorithm is thus well-posed.

Let us now turn to the energy estimate. At the continuous level, this relation is obtained for the barotropic Navier-Stokes equations by choosing the velocity $\boldsymbol{u}$ as a test function in the variational form of the momentum balance equation, writing the convection term as the time derivative of the kinetic energy, and setting the pressure work, namely $-\int_{\Omega} p \operatorname{div}(\boldsymbol{u}) \mathrm{d} \boldsymbol{x}$, under a convenient form. This latter step is done by the following formal computation. Let $b(\cdot)$ be a regular function from $(0,+\infty)$ to $\mathbb{R}$, and let us multiply the mass balance by $b^{\prime}(\rho)$. Using:

$$
\begin{aligned}
b^{\prime}(\rho) \operatorname{div}(\rho \boldsymbol{u})=b^{\prime}(\rho)[\boldsymbol{u} \cdot \nabla \rho+\rho \operatorname{div}(\boldsymbol{u})]=\boldsymbol{u} \cdot \boldsymbol{\nabla} b(\rho)+\rho b^{\prime}(\rho) \operatorname{div}(\boldsymbol{u}) & \\
& =\operatorname{div}(b(\rho) \boldsymbol{u})+\left[\rho b^{\prime}(\rho)-b(\rho)\right] \operatorname{div}(\boldsymbol{u}),
\end{aligned}
$$

we get:

$$
\partial_{t}[b(\rho)]+\operatorname{div}[b(\rho) \boldsymbol{u}]+\left[\rho b^{\prime}(\rho)-b(\rho)\right] \operatorname{div}(\boldsymbol{u})=0 .
$$

Choosing now the function $b(\cdot)$ in such a way that $\rho b^{\prime}(\rho)-b(\rho)=\wp^{-1}(p)$, integrating over $\Omega$ and supposing homogeneous Dirichlet boundary conditions over $\partial \Omega$ yields:

$$
-\int_{\Omega} p \operatorname{div}(\boldsymbol{u}) \mathrm{d} \boldsymbol{x}=\frac{d}{d t} \int_{\Omega} b(\rho) \mathrm{d} \boldsymbol{x} .
$$

The following lemma [7] states a discrete counterpart of this computation.

Lemma 4.1 Let us suppose that the velocity field obeys homogeneous Dirichlet boundary conditions. Let $b(\cdot)$ be a regular convex function from $(0,+\infty)$ to $\mathbb{R}$, and $\left(\rho_{K}^{\star}\right)_{K \in \mathcal{M}}$ be a positive family of real numbers. Then, with the upwind discretization (3) of the mass balance equation, the family $\left(\rho_{K}\right)_{K \in \mathcal{M}}$ is also positive, and we get:

$$
\begin{aligned}
\sum_{K \in \mathcal{M}} b^{\prime}\left(\rho_{K}\right)\left[\frac{|K|}{\delta t}\left(\rho_{K}-\rho_{K}^{*}\right)+\sum_{\sigma \in \mathcal{E}(K)} F_{K, \sigma}\right] \geq & \\
& \frac{1}{\delta t} \sum_{K \in \mathcal{M}}|K|\left[b\left(\rho_{K}\right)-b\left(\rho_{K}^{*}\right)\right]+\sum_{K \in \mathcal{M}}\left[\rho_{K} b^{\prime}\left(\rho_{K}\right)-b\left(\rho_{K}\right)\right] \sum_{\sigma \in \mathcal{E}(K)}|\sigma| \boldsymbol{u}_{\sigma} \cdot \boldsymbol{n}_{K, \sigma} .
\end{aligned}
$$

We are now in position to state the following stability result.

TheOREM 4.2 Let us suppose that the velocity field obeys homogeneous Dirichlet boundary conditions. The scheme (14)-(16) satisfies the following energy identity, for $1 \leq n \leq N$ :

$$
\begin{aligned}
\frac{1}{2} \sum_{i=1}^{d} \sum_{\sigma \in \mathcal{E}_{\text {int }}^{(i)}}\left|D_{\sigma}\right| \rho_{\sigma}^{n-1}\left(\boldsymbol{u}_{\sigma, i}^{n}\right)^{2}+\delta t \sum_{k=1}^{n} \sum_{\sigma \in \mathcal{E}^{(i)}}\left|D_{\sigma}\right|\left(\operatorname{div} \tau\left(\boldsymbol{u}^{k}\right)\right)_{\sigma}^{(i)} \boldsymbol{u}_{\sigma, i}^{k} \\
+\sum_{K \in \mathcal{M}}|K| b\left(\rho_{K}^{n}\right) \leq \frac{1}{2} \sum_{i=1}^{d} \sum_{\sigma \in \mathcal{E}_{\text {int }}^{(i)}}\left|D_{\sigma}\right| \rho_{\sigma}^{(-1)}\left(\boldsymbol{u}_{\sigma, i}^{0}\right)^{2}+\sum_{K \in \mathcal{M}}|K| b\left(\rho_{K}^{0}\right) .
\end{aligned}
$$

The proof of this theorem is based on Lemma 3.1 and Lemma 4.1, and may be found, for the essential arguments, in [7]. 

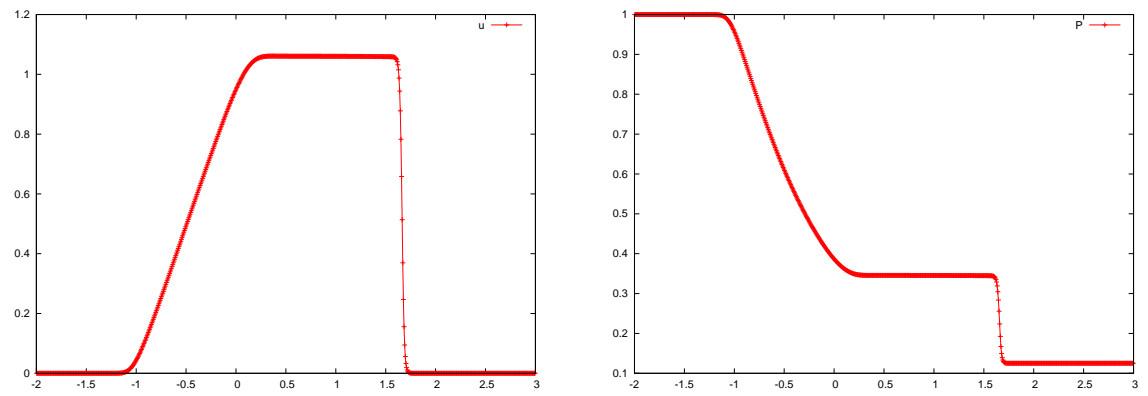

Figure 4: Solution for the Sod shock-tube problem, obtained with a uniform mesh of 800 cells, with a residual viscosity - left: velocity, right: pressure.

Remark 3 Let us suppose that the equation of state reads $p=\rho^{\gamma}$, with $\gamma \in(1,+\infty)$. Then an easy computation yields $b(\rho)=\rho^{\gamma} /(\gamma-1)=p /(\gamma-1)$. Theorem 4.2 thus yields an estimate for the pressure in $\mathrm{L}^{\infty}\left(0, T ; \mathrm{L}^{1}\right)$-norm. Note that this estimate is however not sufficient to ensure that a sequence of pressures obtained as discrete solutions converges to a function; in fact, in convergence studies of numerical schemes $[8,5,6]$ as well as in mathematical analysis of the continuous problem [21], the pressure has to be controlled from estimates of its gradient.

\section{Euler equations and solutions with shocks}

In this section we briefly discuss the capability of the considered numerical schemes to compute irregular (i.e. with discontinuities) solutions of inviscid flows.

The results obtained with the above described pressure correction scheme for the so-called onedimensional Sod shock-tube problem are displayed on Figure 4 (see [19] for a more detailed presentation). From numerical experiments, it seems that this scheme converges when the velocity space translates are controlled, either by upwinding the discretization of the velocity convection term, or by keeping a residual viscosity in the (discrete) momentum balance equation. Numerical experiments reported in [19] (addressing also an extension of this algorithm to the barotropic homogeneous two-phase flow model [11]) confirm the stability of the scheme, and show that the qualitative behaviour of the solution is captured up to very large values of the CFL number (typically, in the range of 50).

From the theoretical point of view, for Euler equations (i.e., precisely speaking, with a diffusion vanishing with the space step), the control that we are able to prove on the solution of course does not yield (weak or strong) convergence in strong enough norms to pass to the limit in the scheme. We can however prove the following result: supposing convergence for the density in $\mathrm{L}^{p}(\Omega), p \in[1,+\infty)$ and for the velocity in $\mathrm{L}^{r}(\Omega), r \in[1,3]$, it is possible to pass to the limit in the discrete equations, provided that the viscosity vanishes as $h^{\alpha}, \alpha \in(0,2)$ for both the implicit and the pressure correction scheme. In this case, the limit of a sequence of discrete solutions is proven to satisfy the weak form of the Euler equations, and so, in particular, the Rankine-Hugoniot conditions at the shocks. 


\section{Discussion and perspectives}

The theoretical analysis of the schemes presented here has been undertaken for model stationary problems: in $[8,5]$, we prove the convergence for the Crouzeix-Raviart discretization of the Stokes equations (with the additional stabilization term needed for purely technical reasons); in [6], we prove the same result for the (standard) MAC scheme. An extension, still for the MAC discretization, to the stationary Navier-Stokes equations is underway.

From a practical point of view, a next step for the barotropic Navier-Stokes equations should be to derive an upwind explicit version of the scheme presented here; in this direction, an extension of Lemma 3.1 (stability of the velocity convection term) to the explicit case may be found in [9].

The main objective is however to deal with the full (i.e. non barotropic, therefore including an energy balance) Navier-Stokes equations. An unconditionally stable pressure correction scheme has been derived for this problem [17], but extensive tests of this scheme remain to be done. In particular, stability requires that the internal energy remains non-negative (in practice, positive); the way we obtained this property was to solve the internal energy balance, with a scheme able to preserve the sign of the unknown. However, it is commonly agreed that, for the scheme to converge toward the correct weak solution, a conservative discretization of the total energy balance should be used. The actual occurrence of this problem, and the possibility to circumvent it, possibly by adding stabilizing viscous terms, will deserve investigations in the near future; a preliminary step on this route may be found in [10].

\section{References}

[1] G. Ansanay-Alex, F. Babik, J.-C. Latché, D. Vola: An $\mathrm{L}^{2}$-stable approximation of the Navier-Stokes convection operator for low-order non-conforming finite elements. IJNMF, online (2010).

[2] C.H. Bruneau, P. Fabrie: Effective downstream boundary conditions for incompressible Navier-Stokes equations. IJNMF, 99, 693-705 (1994).

[3] M. Crouzeix, P.-A. Raviart: Conforming and nonconforming finite element methods for solving the stationary Stokes equations I, Revue Française d'Automatique, Informatique et Recherche Opérationnelle (R.A.I.R.O.), R-3, 33-75 (1973).

[4] F. Dardalhon, J.-C. Latché, S. Minjeaud: Analysis of a projection method for low-order non-conforming finite elements. Submitted (2011).

[5] R. Eymard, T. Gallouët, R. Herbin, J.-C. Latché: A convergent Finite Element-Finite Volume scheme for the compressible Stokes problem. Part II: the isentropic case. Mathematics of Computation, 79, 649-675 (2010).

[6] R. Eymard, T. Gallouët, R. Herbin, J.-C. Latché: Convergence of the MAC scheme for the compressible Stokes equations. SIAM Journal on Numerical Analysis, 48, 2218-2246 (2010).

[7] T. Gallouët, L. Gastaldo, R. Herbin, J.-C. Latché: An unconditionally stable pressure correction scheme for compressible barotropic Navier-Stokes equations. Mathematical Modelling and Numerical Analysis, 42, 303-331 (2008). 
[8] T. Gallouët, R. Herbin, J.-C. Latché: A convergent Finite Element-Finite Volume scheme for the compressible Stokes problem. Part I: the isothermal case. Mathematics of Computation, 78, 1333-1352 (2009).

[9] T. Gallouët, R. Herbin, J.-C. Latché: Kinetic energy control in explicit Finite-Volume discretizations of the incompressible and compressible Navier-Stokes equations. International Journal of Finite Volumes 2 (2010).

[10] T. Gallouët, R. Herbin, J.-C. Latché, T.T. Nguyen: Playing with Burgers equation. Finite Volumes for Complex Applications VI (FVCA VI), these proceedings.

[11] L. Gastaldo, R. Herbin, J.-C. Latché: An unconditionally stable Finite Element-Finite Volume pressure correction scheme for the drift-flux model. Mathematical Modelling and Numerical Analysis, 44, 251-287 (2010).

[12] J.-L. Guermond, L. Quartapelle: A Projection FEM for Variable Density Incompressible Flows. Journal of Computational Physics, 165, 167-188 (2000).

[13] F.H. Harlow, J.E. Welsh: Numerical calculation of time-dependent viscous incompressible flow of fluid with free surface. Physics of Fluids, 8, 2182-2189 (1965).

[14] F.H. Harlow, A.A. Amsden: A numerical fluid dynamics calculation method for all flow speeds. Journal of Computational Physics, 8, 197-213 (1971).

[15] R. Herbin, J.-C. Latché: A kinetic energy control in the MAC discretization of compressible Navier-Stokes equations. International Journal of Finite Volumes 2 (2010).

[16] R. Herbin, J.-C. Latché, B. Piar: A finite-element finite-volume face centred scheme with non-conforming local refinement. I - Convection-diffusion equation. In preparation (2011).

[17] R. Herbin, W. Kheriji, J.-C. Latché: An unconditionally stable pressure correction scheme for the compressible Navier-Stokes equations. In preparation (2011).

[18] ISIS: a CFD computer code for the simulation of reactive turbulent flows, https://gforge.irsn.fr/gf/project/isis.

[19] W. Kheriji, R. Herbin, J.-C. Latché: Numerical tests of a new pressure correction scheme for the homogeneous model. ECCOMAS CFD 2010, Lisbon, Portugal, June 2010.

[20] C. Lapuerta, J.-C. Latché: Discrete artificial boundary conditions for compressible external flows. In preparation (2011).

[21] P.-L. Lions: Mathematical Topics in Fluid Mecanics. Volume 2. Compressible Models. Oxford Lecture Series in Mathematics and its Applications, vol. 10 (1998).

[22] PELICANS: Collaborative Development Environment. https://gforge.irsn.fr/gf/project/pelicans.

[23] R. Rannacher, S. Turek: Simple Nonconforming Quadrilateral Stokes Element. Numerical Methods for Partial Differential Equations, 8, 97-111 (1992). 